\title{
CLINICAL APPLICATION OF SPHARM-PDM TO QUANTIFY TEMPOROMANDIBULAR JOINT OSTEOARTHRITIS
}

\author{
Beatriz Paniagua $^{\mathrm{a}}$, Lucia Cevidanes ${ }^{\mathrm{a}}$, David Walker ${ }^{\mathrm{a}}$, Hongtu Zhu ${ }^{\mathrm{b}}$, Ruixin Guo ${ }^{\mathrm{b}}$, and Martin \\ Styner ${ }^{\mathrm{C}}$ \\ aDepartment of Orthodontics, University of North Carolina at Chapel Hill \\ bDepartment of Biostatistics, University of North Carolina at Chapel Hill \\ 'Department of Computer Science and Psychiatry, University of North Carolina at Chapel Hill
}

\begin{abstract}
The severe bone destruction and resorption that can occur in Osteoarthritis of the Temporomandibular Joint (TMJ) is associated with significant pain and limited joint mobility. However, there is no validated method for the quantification of discrete changes in joint morphology in early diagnosis or assessment of disease progression or treatment effects. To achieve this, the objective of this cross-sectional study was to use simulated bone resorption on cone-beam CT (CBCT) to study condylar morphological variation in subjects with temporomandibular joint (TMJ) osteoarthritis (OA). The first part of this study assessed the hypothesis that the agreement between the simulated defects and the shape analysis measurements made of these defects would be within $0.5 \mathrm{~mm}$ (the image's spatial resolution). One hundred seventy-nine discrete bony defects measuring $3 \mathrm{~mm}$ and $6 \mathrm{~mm}$ were simulated on the surfaces of 3D models derived from CBCT images of asymptomatic patients using ITK-Snap software. SPHARM shape correspondence was used to localize and quantify morphological differences of each resorption model with the original asymptomatic control. The size of each simulated defect was analyzed and the values obtained compared to the true defect size. The statistical analysis revealed very high probabilities that mean shape correspondence measured defects within $0.5 \mathrm{~mm}$ of the true defect size. $95 \%$ confidence intervals (CI) were $(2.67,2.92)$ and $(5.99,6.36)$ and $95 \%$ prediction intervals (PI) were $(2.22,3.37)$ and $(5.54,6.82)$, respectively for $3 \mathrm{~mm}$ and $6 \mathrm{~mm}$ simulated defects. The second part of this study applied shape correspondence methods to a longitudinal sample of TMJ OA patients. The mapped longitudinal stages of TMJ OA progression identified morphological variants or subtypes, which may explain the heterogeneity of the clinical presentation. This study validated shape correspondence as a method to precisely and predictably quantify $3 \mathrm{D}$ condylar resorption.
\end{abstract}

\section{Keywords}

TMJ-OA; SPHARM-PDM; Bone Tissue Reabsorption Simulation; Bone Tissue Reabsorption Measurement

(C) 2010 Elsevier Ltd. All rights reserved.

Conflicts of Interest Statement The study sponsor had no involvement in the study design, in the collection, analysis and interpretation of data; in the writing of the manuscript; and in the decision to submit the manuscript for publication.

Publisher's Disclaimer: This is a PDF file of an unedited manuscript that has been accepted for publication. As a service to our customers we are providing this early version of the manuscript. The manuscript will undergo copyediting, typesetting, and review of the resulting proof before it is published in its final citable form. Please note that during the production process errors may be discovered which could affect the content, and all legal disclaimers that apply to the journal pertain. 


\section{Introduction}

Temporomandibular disorders (TMD) involve the TMJ, muscles of mastication, or both (American Association of Dental Research, 1966). TMD that affects approximately 10\% of the population, is more prevalent in women, and causes pain, crepitus, and bone resorption that leads to functional disabilities and limitations (Helenius et al., 2006). Wiese et al. (Wiese et al., 2008) reported that $42.6 \%$ of TMD patients presented radiographic findings of osteoarthritic changes, i.e., bone erosions and osteophytes in TMJ tomograms (figure 1). Developing effective medical treatments to prevent or to reduce progression of bone damage is hampered by the lack of imaging biomarkers to assess disease progression and the effect of treatment interventions. Current Osteoarthritis (OA) treatments target only clinical manifestations such as significant functional limitations and facial pain and changes in jaw relation with anterior open bite and impaired chewing ability. There is a substancial oportunity now to use TMJ OA as a model in which better measurement of the effect of clinical interventions can lead to further therapeutic approaches with broad application to OA.

Cone-beam computed tomography (CBCT) is a rapidly advancing area of imaging with the capability to provide three dimensional images of hard and soft tissue structures. Even though analysis of cross-sectional CT or CBCT slices can be used to diagnose condylar resorption on patients with TMJ OA, new methods to measure and localize radiologic abnormality in bony tissue are needed to diagnose and assess progression and treatment effects in TMJ OA. (Cevidanes et al., 2007a) (Cevidanes et al., 2007c) (Cevidanes et al., 2006) (Cevidanes et al., 2007b) and currently available quantitative measurements approaches do not support longitudinal clinical evaluations. The need for 3D morphometric methods to analyze the configuration of the joint morphology has been discussed for decades (Christensen et al., 1996) (Rueckert et al., 1999). Current quantitative methods have included volume, score and classification systems, landmark-based measurements, and pairwise surface measurements via Iterative Closest Point. Volumetric (Joe et al., 2007) (Siegel et al., 2007) (Goo et al., 2005) (Thompson et al., 1997) (Levine et al., 2008) changes have excellent applications for certain medical problems, but not for osteoarthritis, since they reflect global changes in size, but do not detect structural changes at specific locations. Correct use of score and classification systems such as the Kellgren and Lawrence (for knee OA) (Peterfy et al., 2008) and Koyama et al. (Koyama et al., 2007) (for TMJ OA) is difficult, and major OA cohort studies disagree between each other and even among themselves on the definition and grading of disease according to these systems (Langs et al., 2009) (Wirth and Eckstein, 2008). Landmark-based (Rohr, 2001) measurements present errors related to landmark identification. Gunz et al. (Gunz et al., 2004) and Andresen et al. (Andresen et al., 2000) proposed the use of semi-landmarks, i.e., landmarks plus vectors and tangent planes that define their location, but information from the whole curves and surfaces must also be included. As noted by those authors, landmarks regardless of how well defined or free of error, are abstractions since information on the spaces, curves, or surfaces between landmarks is lost. Iterative Closest Point (Gerig et al., 2001a) (ICP) is the approach used by most commercial and academic software tools for assessments of differences over time. ICP measures closest distances among pairwise surfaces, which are often based on anatomically incorrect correspondent locations. This happens specially in highly curved anatomical structures such as the mandible. For this reason, ICP fails to appropriately quantify physiologic adaptations such as condylar bone remodeling.

Shape analysis has become of increasing interest to the Medical Image Analysis community due to its potential to precisely locate and quantify morphological changes between healthy and pathological structures. As part of the National Alliance for Medical Image Computing (http://www.namic.org), the Neuro Image Research and Analysis Lab (NIRAL) at the 
University of North Carolina developed a comprehensive set of tools for the computation of 3D structural statistical shape analysis that have been mostly used for brain morphometry studies. The objectives of this study were to test the accuracy of SPHARM-PDM toolbox to assess 3D condylar morphology and to apply these techniques to measure bony changes in TMJ OA.

\section{Methods}

In summary, we performed two separate studies to assess how shape analysis can be used in TMJ. The first study focused on a simulation of bone resorption to validate the methodology and second study was evaluating the method on real datasets of actual TMJ OA patients. For the bone resorption simulation study in this paper, this retrospective cross-sectional study utilized 3D virtual surface models of the mandibular condyles from CBCT images of 20 asymptomatic patients (mean age $=23.7$ years, $\mathrm{SD}=10.7$ years) who sought treatment at the Orthodontic Clinic. For the clinical application study, we assessed CBCT images of 26 jaw surgery patients (mean age $=25.4$ years, $\mathrm{SD}=9.4$ years) with radiographic diagnosis of TMJ OA (Ahmad et al., 2009) who sought treatment at the Orthodontic Clinic were assessed. Imaging was performed presurgery, at splint removal, 1 year and 2 years postsurgery. Biomedical Institutional Review Board approval was obtained for analysis of all the CBCTs. After radiological interpretation, the general and modular methodological framework used for shape analysis of bone resorption models consisted of the following steps (figure 2): (1) segmentation of the CBCT volumes, (2) validation of the segmentation of condylar models, (3) point-based correspondent models calculation using SPHARMPDM correspondence optimization, and (4) statistical analysis. This pipeline was applied to both the bone resorption simulation study and the quantification of joint bone resorption in actual TMJ OA patients.

\subsection{Segmentation of anatomic structures}

Segmentation of the anatomic structures was performed manually, i.e., outlining the structures visible in the cross-sections of a volumetric dataset acquired with the NewTom CBCT-3G (QR-NIM s.r.l., Verona, Italy), via InsightSNAP (Yushkevich et al., 2006). Three-dimensional virtual models of the mandibular left and right condyles for each subject were built from a set of approximately 300 axial cross-sectional slices for each image, with the voxels reformatted to an isotropic of $0.5 \times 0.5 \times 0.5 \mathrm{~mm}^{3}$. Validation of condylar 3D models constructed from CBCT was performed by two different observers who were trained with images not included in this study. The two observers performed segmentation in 10 TMJ OA volumetric condyle datasets, and systematic differences between observers were assessed by means of Hotelling $T^{2}$ test based on covariance matrices.

\subsection{SPHARM-PDM Shape correspondence}

The UNC SPHARM-PDM shape analysis toolbox Shape Correspondence (Styner et al., 2006) was employed to provide a unique and symmetric point correspondence across all measured surfaces. The correspondence was computed via mapping every point on the condylar 3D surface model to a unique position on the unit sphere (Brechbuhler et al., 1995) followed by generating a uniformly triangulated surface based on this spherical mapping (SPHARM-PDM) (Gerig et al., 2001b) (Figure 3). The segmented 3-dimensional surface models of the condyles are converted into surface meshes (figure $3 \mathrm{a}$.), and a spherical parametrization is computed for the surface meshes using area-preserving and distortionminimizing spherical mapping. The SPHARM description is computed from the mesh and its spherical parametrization (figure $3 \mathrm{~b}$.). Using the rst-order ellipsoid from the spherical harmonic coefcients, the spherical parametrizations are aligned to establish correspondence across all surfaces (figure $3 \mathrm{c}$.). The Spharm description is then sampled into a triangulated 
surfaces (SPHARM-PDM). The condylar surfaces are represented using a subdivision level 10 (1,002 points in the surface of the condyle). Alignment of all surfaces was performed using rigid Procrustes alignment.

\subsection{Statistical Shape Analysis}

Three statistical analyses were used to analyze the accuracy of defect representation using SPHARM-PDM: (1) $P\left(\left|X-\mu_{0}\right|\right)<0.5$, the probability that the sample mean was within $0.5 \mathrm{~mm}$ of the true defect size, (2) $95 \%$ confidence interval (CI), and (3) $95 \%$ prediction interval (PI). All analyses were based on the assumption of a normal distribution for this population.

1. $P\left(\left|\bar{X}-\mu_{0}\right|\right)<0.5$ calculated the probability that the difference between the sample mean $(\bar{X})$ and the hypothesized mean was less than 0.5. If an individual measurement had a $>0.5$ difference from $3 \mathrm{~mm}$ or $6 \mathrm{~mm}$, then the sample mean might not be $3 \mathrm{~mm}$ or $6 \mathrm{~mm}$.

2. $95 \%$ CI: $95 \%$ CI provided an interval with $95 \%$ confidence that true mean of $d$ fell within the interval. Based on the sample information, the CI was obtained by

$\bar{X} \pm t_{\left(0.025, n_{d}-1\right)} \frac{s_{d}}{\sqrt{n_{d}}}$ where $t_{\left(0.025, n_{d}-1\right)}$ is the critical value of the $\mathrm{t}$-distribution with $d f=n_{d}-1$. The confidence interval did not have to contain the true mean since it measured the probability that the intervals contain the true mean. In other words, if 100 samples were drawn and its CIs calculated, then approximately 95 CIs would contain the true mean.

3. $95 \%$ PI provided an estimate of an interval that a future observation of a random variable (if any) would fall within the range of the interval. It can be considered as a confidence interval for prediction. The prediction interval is always wider than the confidence interval because of the additional uncertainty for prediction. PI can be computed by $\bar{X} \pm t_{\left(0.025, n_{d}-1\right)} \sqrt{1+\frac{1}{n_{d}}}$.

\subsection{Bone resorption}

In order to assess the validity of the proposed methodological framework, 179 discrete bony resorption defects were generated for 20 right and left condyles. In the next subsections, the steps involved in this simulation study will be explained in detail.

2.4.1. Simulation-The bone resorption defects were simulated by a human expert by marking resorption areas in the binary segmentations of healthy condyles (see Figure 4). The following defects were simulated, labeled by location and magnitude of the defect: $3 \mathrm{C}(3 \mathrm{~mm}$ depth defect in the central part of the condyle), $3 \mathrm{M}$ (3mm depth defect in the medial part of the condyle), 3L ( $3 \mathrm{~mm}$ depth defect in the lateral part of the condyle), 3CML ( $3 \mathrm{~mm}$ depth defects in the medial, central and lateral part of the condyle), and 6C (6mm depth defect in the central part of the condyle). Table 1 details the total simulated data in this study.

2.4.2. Defect Measurement-Shape differences were calculated between each resorption $3 \mathrm{D}$ model and the correspondent original 3D models. These differences were visualized with color-coded magnitude maps and difference vector maps (see figure 5). We selected designated region of interests (ROI) for each type of simulation that select affected regions for further study. Difference magnitude maps were masked using the ROIs via the following steps: 
1. Calculation of absolute difference maps between each resorption $3 \mathrm{D}$ model and the original 3D model.

2. Selection of ROIs, i.e., selection of all points within each simulated defect in the condyle. For models with more than one defect (i.e. 3CML), an ROI was determined for each defect.

3. Calculation of each ROI absolute differences map, using the ROI as a mask. This step generates a map a using the outcome in step 1 that only shows distances for the points in the selected ROI (step 2).

4. The depth of each bone defect was measured via the surface point with the maximum difference in the ROI difference and its six neighboring triangles. We compute the depth as the average over the distances of these triangles, with each triangle distance computed by averaging its vertices' distances (triangle value $=$ $\left(\right.$ point $_{0}+$ point $_{1}+$ point $\left._{2}\right) / 3$.

\subsection{Longitudinal Analysis}

Given the favorable results obtained in the simulation study, this morphological framework was used for assessing disease progression in TMJ OA. Measurements of longitudinal changes including 3D absolute distances, signed distances and vectors of bone changes were obtained for each condyle. Shape differences were calculated between baseline 3D model and one year and two year follow-up 3D models. Rotational displacements and regions of surface remodeling in the condylar area were visualized with color-coded magnitude maps and difference vector maps (see Figures 9 and 10). In addition, three-dimensional virtual surface models allowed clear visualization of morphological characteristics of osteoarthritic TMJ, i.e., surface erosions, osteophytes and surface flattening (see Figures 7 and 8).

\section{Results}

\subsection{Validation of condylar 3D models}

Small interrater variability with mean distance $0.64 \mathrm{~mm} / 0.68 \mathrm{~mm}$ and maximum distance $1.11 \mathrm{~mm} / 1.13 \mathrm{~mm}$ were found between the composite models from each observer respectively for left and right condyles. Raw significance maps showed no significant interobserver differences (all points $p>0.05$ ). Figure 6 shows semitransparent overlays of the average models for left and right condyles, demonstrating that no relevant differences between observers exist.

\subsection{Bone Resorption Simulation}

The results for right and left condyles grouping are given in Table 2, and for defects grouping in Table 3. Probabilities, 95\% CI and 95\% PI for quantification of bone defects of different sizes, simulated at different anatomic locations in the mandibular condyles, are shown in each table.

In Table 2, the column for 3CML shows the average statistics for those three defects. Statistical analysis results revealed very high probability (ranging 0.93-1) that the difference between the sample hypothesized mean ( 3 or $6 \mathrm{~mm}$ defect) was less than $0.5 \mathrm{~mm}$. In Table 2 all obtained values for $P\left(\left|\bar{X}-\mu_{0}\right|\right)<0.5$ left condyles were high (ranging 0.998-1). For this clinical application, measurements within the spatial resolution of the image (in this case $0.5 \mathrm{~mm}$ ) were considered accurate. The results showed an acceptable error range in measurements calculated for the left condyles with SPHARM correspondence. The measurements calculated for $6 \mathrm{~mm}$ defects in the right condyles showed the lowest probability $(0.93$, see Table 2 , last column). However, all cases showed $>0.9$ probability, 
which is still high probability. The $95 \%$ CI tests contained the hyphotesized means $(\bar{X}=3$ or $X=6$ ), except for $3 \mathrm{~mm}$ defects on the right condyles. All the 95\% PI intervals for left and right condyles contained the hyphotesized means (Observe that PI is wider than CI, see Table 2). Table 3 also showed positive results, since the grouping made here was not based on the place the defect was generated (right or left condyle), but was based on the size. $P(\mid X$ $\left.-\mu_{0} \mid\right)<0.5$ is 1 for both $3 \mathrm{~mm}$ and $6 \mathrm{~mm}$ defects, showing that all the measured defects would be within $0.5 \mathrm{~mm}$ of the simulated defect size. The $95 \%$ CI for $3 \mathrm{~mm}$ defect did not contain $X=3$, however this interval was very close to this value $(2.67,2.92)$. Both $95 \%$ PI contained respective hyphotesized means.

\subsection{Clinical case example of longitudinal asssements of condylar resorption}

Patient presented in the clinic with severe open bite that developed as a sequel of marked bone resorption in the mandibular condyles between before and 2 years after jaw surgery. This patient received maxillary impaction for correction of open bite. The baseline CBCT taken at age 19.5 y showed radiographic signs of generalized sclerosis and flattening on both right and left condyles, confirming the radiographic diagnosis of TMJ OA. Condylar flattening was present in both right and left condyles before surgery, and was more severe on the right condyle. Condylar flattening progressed during postsurgery orthodontics and after treatment. Registration and semitransparent overlays shows condylar changes between pre-surgery (transparent mesh lines) and recall (surface models). Right lateral views of jaw surface models from pre-surgery through recall 2 years postsurgery ( 1 year post orthodontics) show the general mandibular anatomy of the patient across time. Frontal views of condylar morphology prior to surgery through recall C. Registration and overlay of show progression of condylar changes (see Figure 8).

Measurements of longitudinal changes including 3D absolute distances, signed distances and vectors of bone changes were obtained for each condyle (see figures 9 and 10). Close up views of the longitudinal follow-up of changes in the right and left condyles. Absolute distances and vectors of bone changes are obtained at each of 1002 points in the surface of the condyles. Visualization of changes using signed distances are also provided for better understanding of the bone remodeling (figures 9 and $10 \mathrm{~F}$.).

Right condylar presurgery model subtracted from splint removal model show mild bone remodeling in the superior surface fo the condyle. Splint removal subtracted from 1 year postsurgery shows marked bone resorption in the medial superior and anterior aspects of the right condyle; one year post-surgery subtracted from 2 years postsurgery shows continuous condylar bone remodeling particularly of the superior and anterior surfaces of the condyle; finally presurgery subtracted from 2 years showing the marked change in condylar morphology overtime.

Presurgery left condylar model subtracted from splint removal model show mild bone remodeling in the superior surface fo the condyle. Splint removal subtracted from 1 year postsurgery left models show mild bone resorption in the superior aspect of the condyle; one year postsurgery subtracted from 2 years postsurgery shows continuous and marked condylar bone remodeling particularly of the superior surface of the left condyle; Presurgery model subtracted from 2 years follow up model showing the marked change in condylar morphology overtime.

\section{Discussion}

The accurate results presented in this study indicate that the SPHARM-PDM toolbox can be used to assess treatment effects and potentially allow future investigations of the association between local morphological changes with clinical and biological markers. The results of the 
shape analysis based quantification in the presented study identified and precisely measured morphological differences and bone breakdown in TMJ OA progression.

Three-dimensional modeling of cone-beam CT imaging has allowed for an increased understanding of the patho-physiological process of TMJ-OA. The methods and results presented in this document provide three dimensional models of various degrees of osteoarthritic remodeling, an assessment of TMJ-OA morphology as compared to asymptomatic morphology, a validation of a novel tool of analyzing discrete OA defects, and show promising results of longitudinal assessments of TMJ-OA morphology. Overall, these studies indicate our next steps towards a more exhaustive investigation of TMJ-OA morphology using CBCT and 3D shape analysis. Our findings reveal the suitability of SPHARM-PDM toolbox to accurately measure condylar resorption in TMD arthritis, and that this toolbox can be used to localize and quantify the degree of change in bone morphology. 3D shape analysis can be also used to relate degree and extent of condylar resorption, and to determine the severity of the disease as a complement to the current diagnostic procedures used in clinical environment. All measurements were in a range of $0.5 \mathrm{~mm}$ of the simulated defect size, which is considered to be an appropiate precision for this clinical application.

Areas for future investigations could focus on prospective analysis and examination of morphologic risk factors and associations with the signs and symptoms of TMJ OA. Given the favorable results in this pilot validation study, our future studies will utilize a framework of morphological (as seen in this manuscript), clinical and biological measurements to establish specific biomarkers for assessing treatment of TMJ OA.

\section{Acknowledgments}

The research was funded by NIDCR DE017727, NIDCR E018962, The UNC NDRC NIH HD 03110, and the NIH Roadmap Grant U54 EB005149-01 (NA-MIC).

\section{References}

Ahmad M, Hollender L, Anderson Q, Kartha K, Ohrbach R, Truelove E. Research diagnostic criteria for temporomandibular disorders ( $\mathrm{rdc} / \mathrm{tmd}$ ): development of image analysis criteria and examiner reliability for image analysis. Oral Surgery, Oral Medicine, Oral Pathology, Oral Radiology and Endodontics. 2009

American Association of Dental Research. Temporomandibulardisorders (tmd). Policy Statement. 1966; 18(4)

Andresen R, Bookstein F, Conradsen K, Ersboll B, Marsh J, Kreiborg S. Surface-bounded growth modeling applied to human mandibles. IEEE Transactions on Medical Imaging. 2000; 1(19):10531063. [PubMed: 11204843]

Brechbuhler C, Gerig G, Kubler O. Parameterization of clsed surfaces for 3d shape description. Computer Vision, Graphics, Image Processing: Image Understanding. 1995

Cevidanes L, Bailey L, Tucker S, Styner M, Mol A, Phillips C, Proffit W, Turvey T. Threedimensional cone-beam computed tomography for assessment of mandibular changes after orthognathic surgery. American Journal of Orthodontics and Dentofacial Orthopedics. 2007a; 1(131):44-50.

Cevidanes L, Oliveira AEF, Phillips C, Motta A, Styner M, Tyndall D. Three dimensional short-term mandibular displacements following class iii surgery. Journal of Dental Research - Special Issue A. 2007b; 1(86): 1827.

Cevidanes L, Phillips C, Lee B, Proffit W. 3d mandibular relapse and remodeling 1 year after orthognathic surgery. 106Th Annual Session of the American Association of Orthodontists. 2006; 1(14):41. 
Cevidanes LHS, Styner M, Phillips C, Oliveira A, Tulloch J. 3d morphometric changes 1 year after jaw surgery accepted biomedical imaging: Macro to nano. IEEE International Symposium in Biomedical Imaging. 2007c; 1(1):1332-1335.

Christensen G, Rabbitt R, Miller M. Deformable templates using large deformation kinematics. IEEE Trans Image Processing. 1996; 1(5):1435-1447.

Gerig G, Jomier M, Valmet C. A new validation tool for assessing and improving $3 \mathrm{~d}$ object segmentation. Proceedings of the International Society and Conference Series on Medical Image Computing and Computer-Assisted Intervention. 2001a; 1(1):516-528.

Gerig G, Styner M, Jones D, Weinberger D, Lieberman J. Shape analysis of brain ventricles using spharm. Mathematical Methods in Biomedical Image Analysis. 2001b; 1:171-178.

Goo J, Tongdee T, Tongdee R, Yeo K, Hildebolt C, Bae K. Volumetric measurement of synthetic lung nodules with multi-detector row ct: effect of various image reconstruction parameters and segmentation thresholds on measurement accuracy. Radiology. 2005; 3(235):850-856. [PubMed: 15914478]

Gunz, P.; Mitteroecker, P.; Bookstein, F. Semilandmarks in three dimensions. Kluwer Academic Publishers; New York: 2004.

Helenius L, Tervahartiala P, Helenius I, Al-Sukhun J, Kivisaari L, Suuronen R. Clinical, radiographic and mri findings of the temporomandibular joint in patients with different rheumatic diseases. International Journal of Oral and Maxillofacial Surgery. 2006; 35:983-989. [PubMed: 17052893]

Joe B, Suh J, Hildebolt C, Hovsepian D, Johnston B, Bae K. Mr volumetric measurements of the myomatous uterus: improved reliability of stereology over linear measurements. Academic Radiology. 2007; 4(14):455-462. [PubMed: 17368215]

Koyama J, Nishiyama H, Hayashi T. Follow-up study of condylar bony changes using helical computed tomography in patients with temporomandibular disorder. Dentomaxillofacial Radiology. 2007; 1(36):472-477.

Langs G, Peloschek P, Bischof H, Kainberger F. Automatic quantification of joint space narrowing and erosions in rheumatoid arthritis. IEEE Transactions on Medical Imaging. 2009; 1(28):151164. [PubMed: 19116197]

Levine B, Kovacevic N, Nica E, Cheung G, Gao F, Schwartz M, Black S. The toronto traumatic brain injury study: injury severity and quantified mri. Neurology. 2008; 10(70):771-778. [PubMed: 18316688]

Peterfy C, Schneider E, Nevitt M. The osteoarthritis initiative: report on the design rationale for the magnetic resonance imaging protocol for the knee. Osteoarthritis Cartilage. 2008; 12(16):14331441. [PubMed: 18786841]

Rohr, K. Landmark-based image analysis: using geometric and intensity models. Kluwer Academic Publishers; Dordrecht, Boston, London: 2001.

Rueckert D, Sonoda L, Hayes C, Hill D, Leach M, Hawkes D. Nonrigid registration using free-form deformations: application to breast mr images. IEEE Trans Med Imaging. 1999; 1(18):712721.

Siegel M, Hildebolt C, Bae K, Hong C, White N. Total and intraabdominal fat distribution in preadolescents and adolescents: measurement with mr imaging. Radiology. 2007; 3(242):846-856. [PubMed: 17244720]

Styner M, Oguz I, Xu1 S, Brechbuhler C, Pantazis D, Levitt J, Shenton M, Gerig G. Framework for the statistical shape analysis of brain structures using spharm-pdm. Insight Journal. July.2006

Thompson P, MacDonald D, Mega M, Holmes C, Evans A, Toga A. Detection and mapping of abnormal brain structure with a probabilistic atlas of cortical surfaces. Journal of Computer Assisted Tomography. 1997; 1(21):567581.

Wiese M, Svensson P, Bakke M, List T, Hintze H, Petersson A. Associations between temporomandibular joint symptoms, signs, and clinical diagnosis using the rdc/tmd and radiographic findings in temporomandibular joint tomograms. Oral Surgery, Oral Medicine, Oral Pathology, Oral Radiology and Endodontics. 2008; 1(22):239-251.

Yushkevich P, Piven J, Hazlett H, Smith R, Ho S, Gee J. User-guided 3d active contour segmentation of anatomical structures: Significantly improved efficiency and reliability. Neuroimage. 2006; 31:1116-1128. [PubMed: 16545965] 
Wirth W, Eckstein F. A technique for regional analysis of femorotibial cartilage thickness based on quantitative magnetic resonance imaging. IEEE Transactions on Medical Imaging. 2008; 6(27): 737-744. [PubMed: 18541481] 


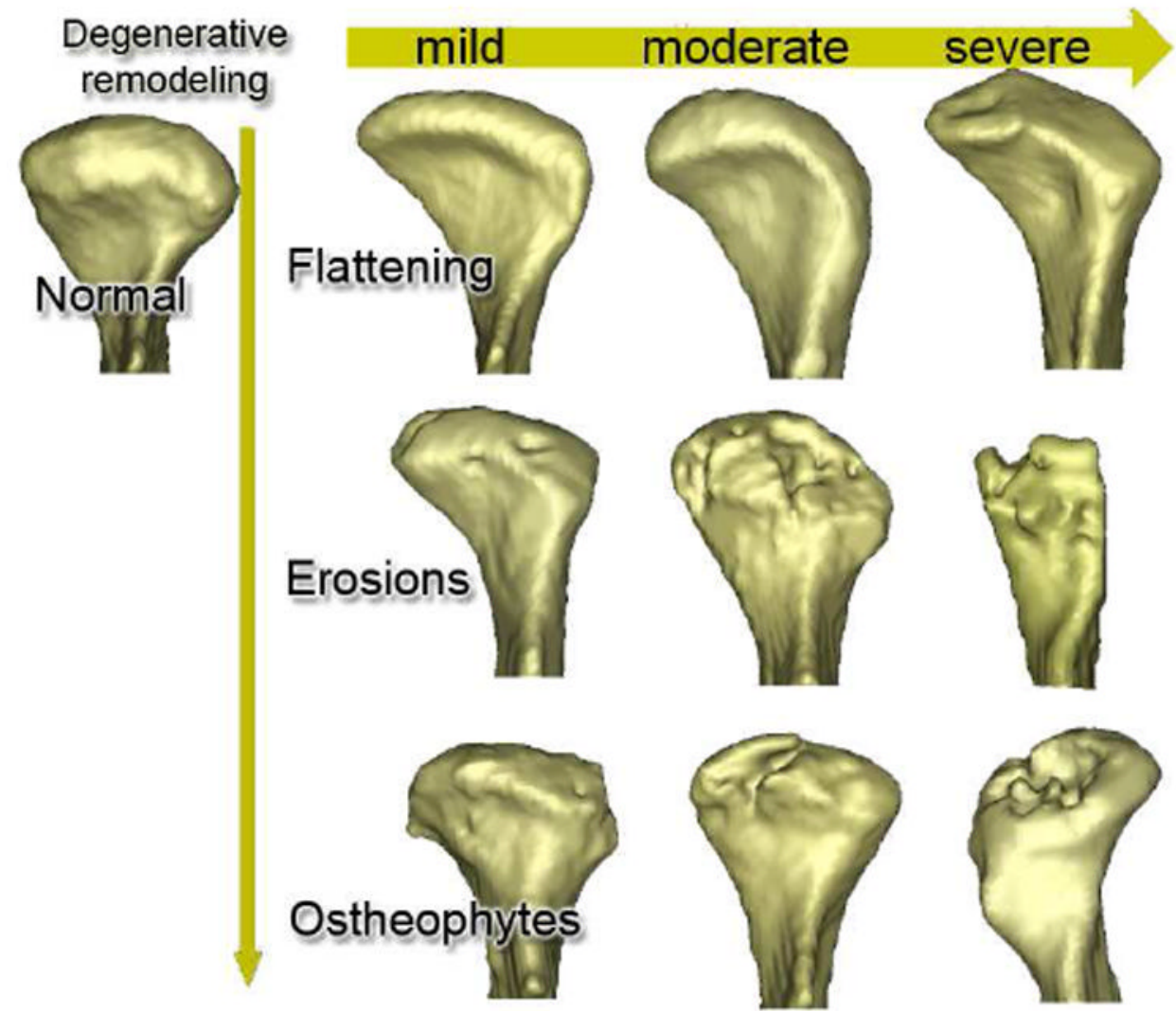

Figure 1.

Spectrum of joint morphology in a cross-sectional study. 


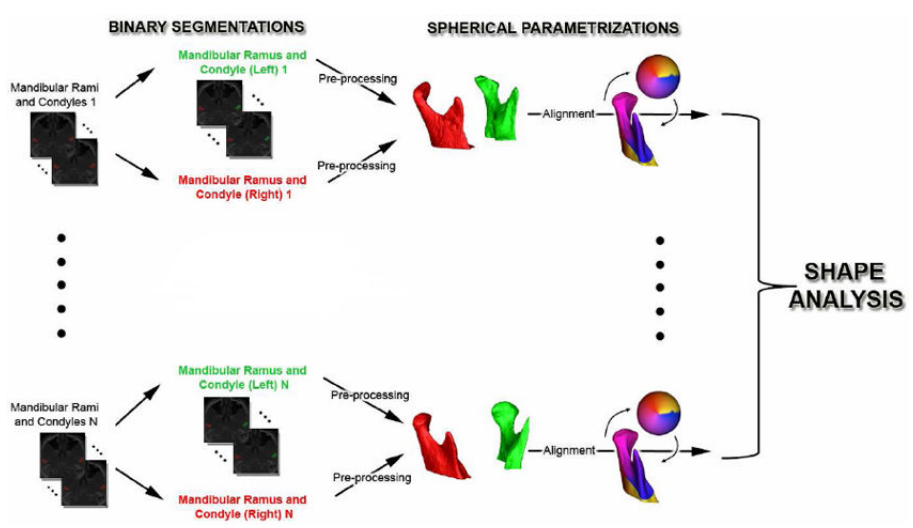

Figure 2.

Workflow used for statistical shape analysis. 


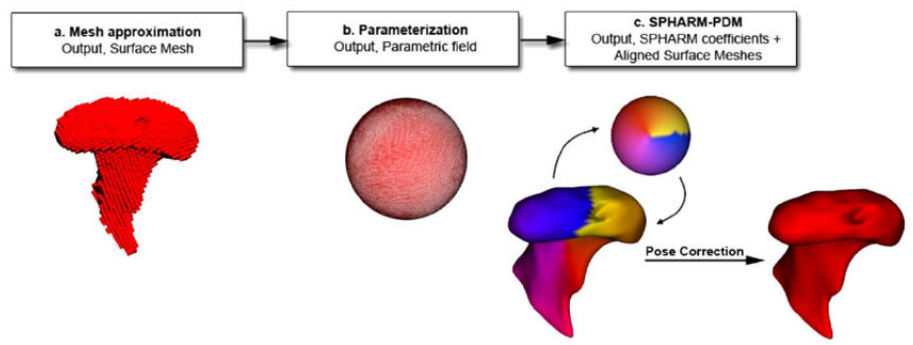

Figure 3.

Description of shape correspondence procedures. 

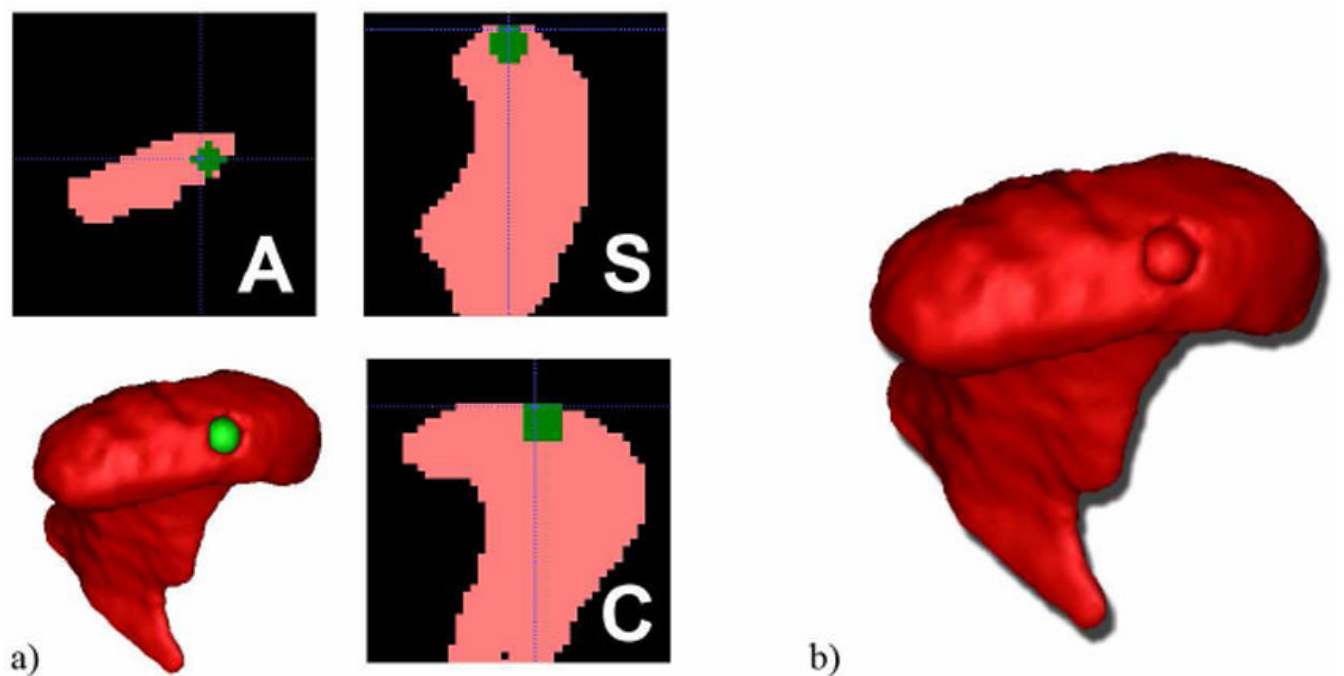

Figure 4.

a) Defect generation in ITK Insight SNAP b) Final result, 3C defect left condyle. 

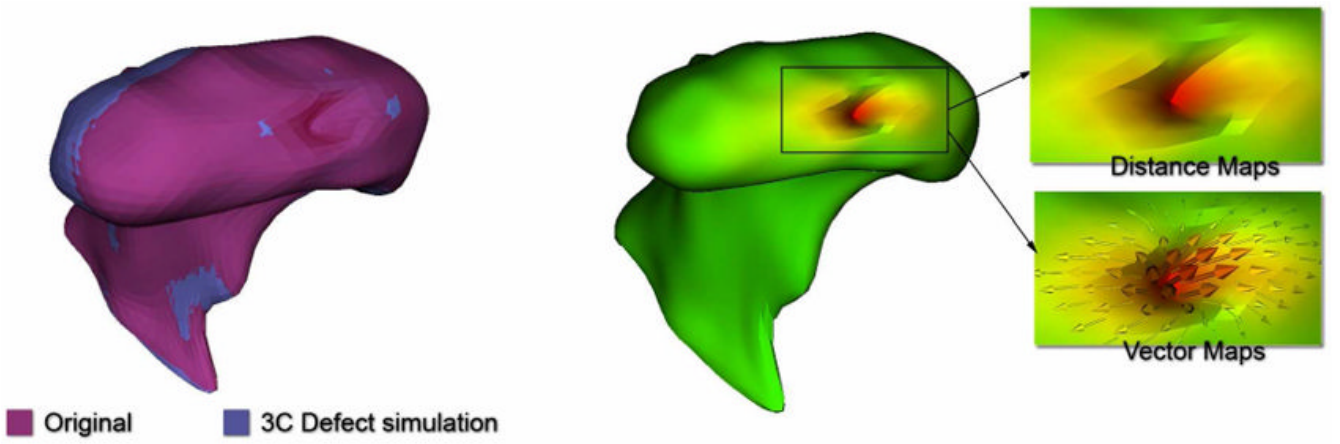

Figure 5.

Example of measurements in a $3 \mathrm{C}$ left condyle. 

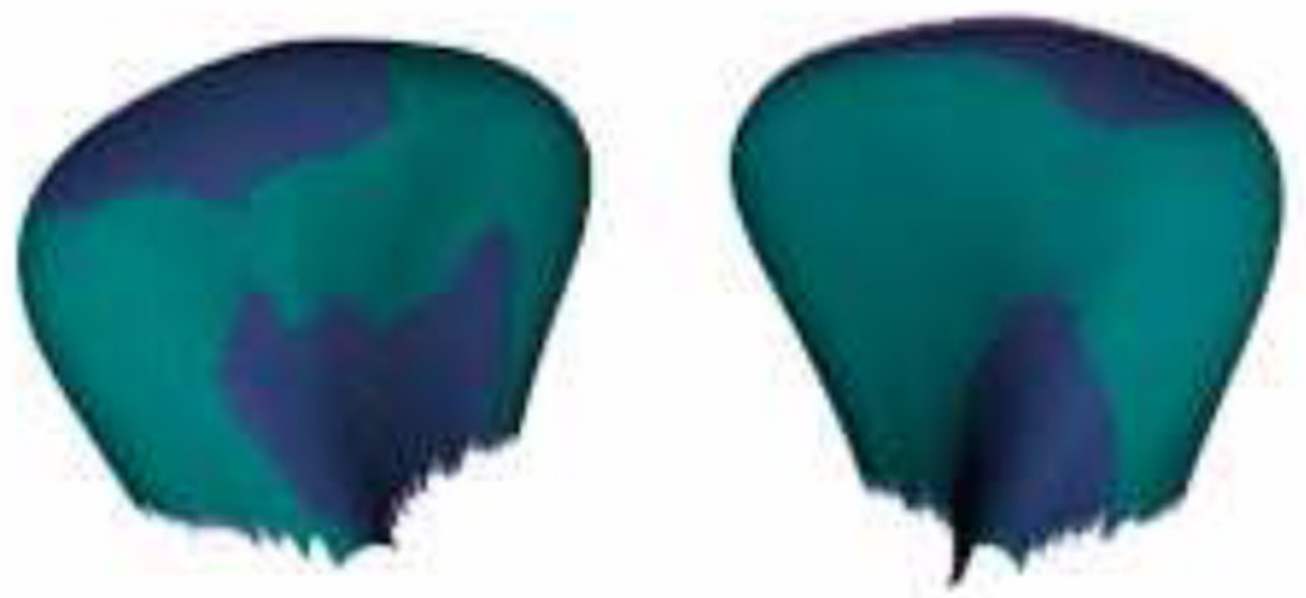

Figure 6.

Semitransparent overlays of average models for each observer (blue and purple) show no significant differences. 

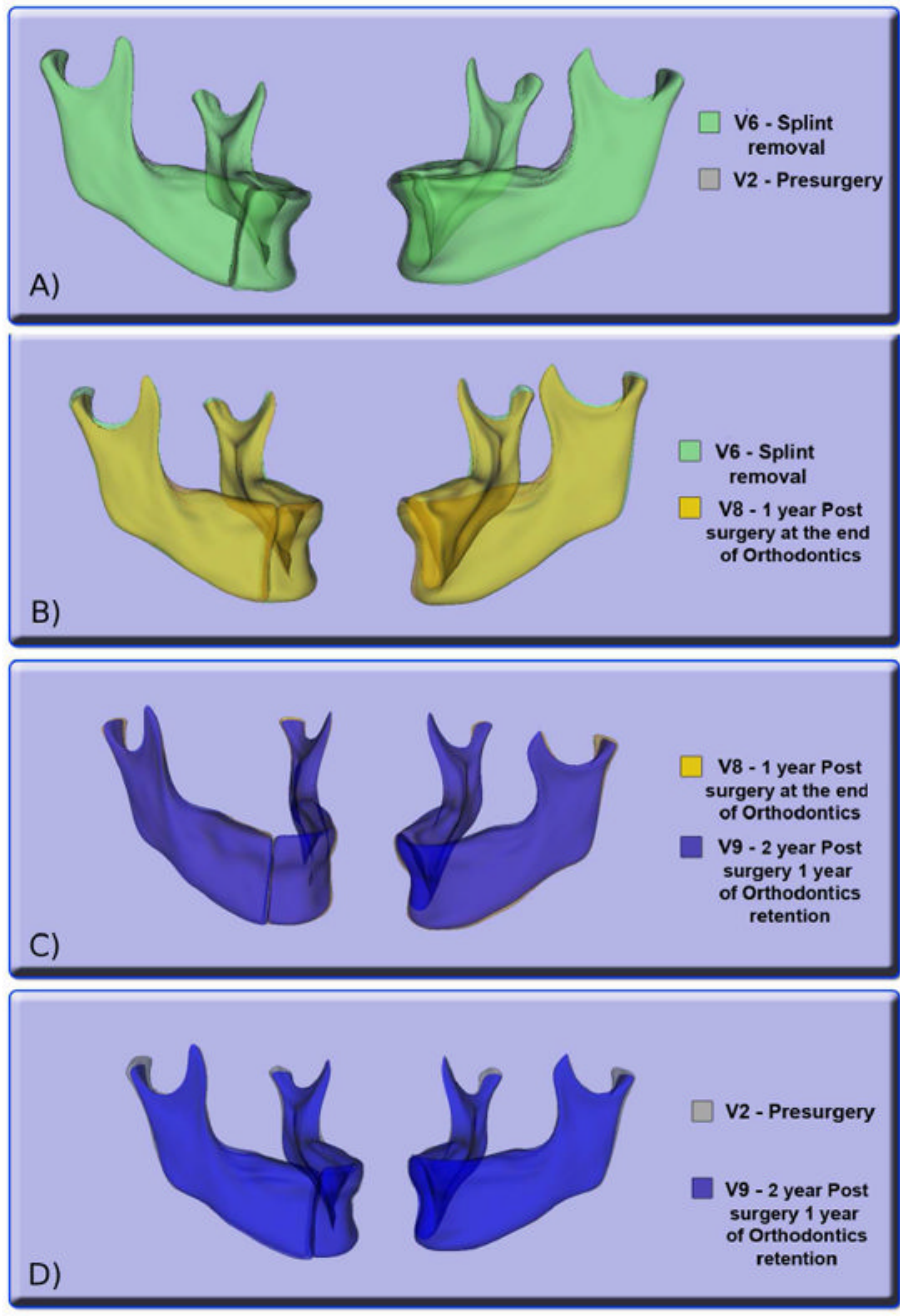

Figure 7.

Surface to surface registration between different time point models allow for accurate longitudinal bone resorption measurement in the condylar area. A. Pre-surgery models compared with models generated after splint removal do not show relevant condylar remodeling. B. One year post surgery models present condylar resorption. C. More marked changes are found when comparing one year post and two years post sugery models. D. Condylar changes from pre surgery to screening two years post surgery. 


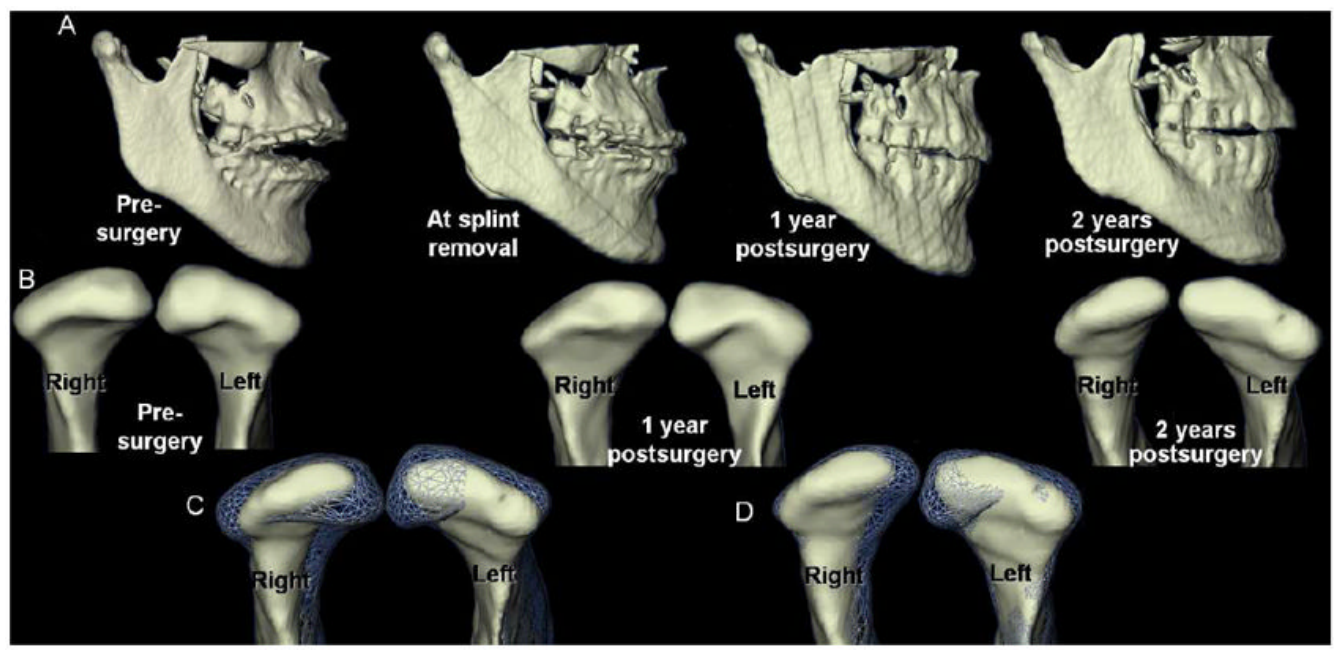

Figure 8.

A. Right lateral views of jaw surface models from pre-surgery through recall 2 years postsurgery (1 year post orthodontics). B. Frontal view of condylar morphology prior to surgery through recall $\mathrm{C}$. Registration and overlay of condylar changes between pre-surgery (transparent mesh lines) and 2 year postsurgery (surface models). D. Registration and overlay show progression of condylar changes between 1 year postsurgery (transparent mesh lines) and 2 year postsurgery (surface models). 
A)
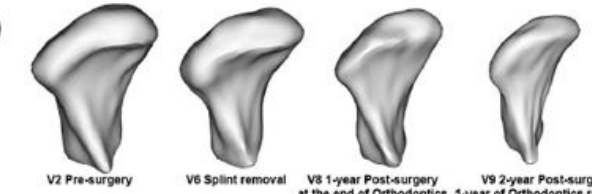

V3 1-year Post-surgery vs 2-year Postsurgery

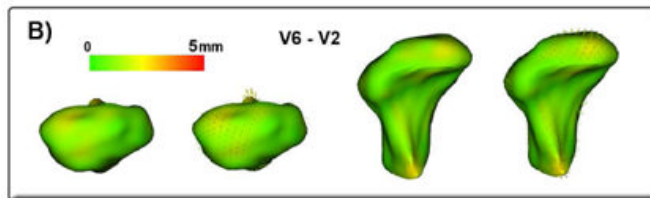

C) $\quad 5 \mathrm{~mm} \quad$ V8 - V6

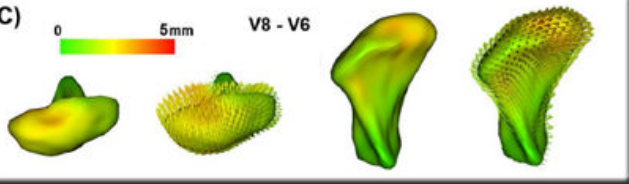

D)

V9 - v8
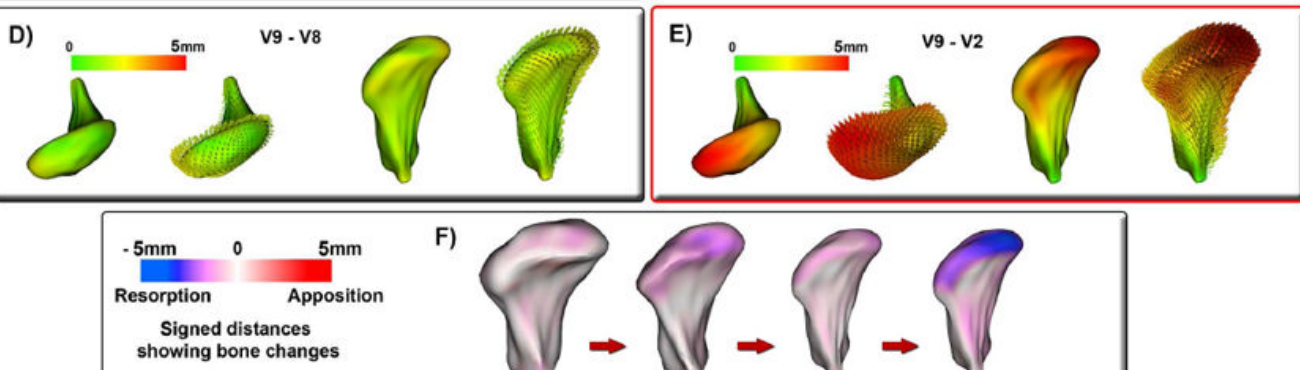

F)

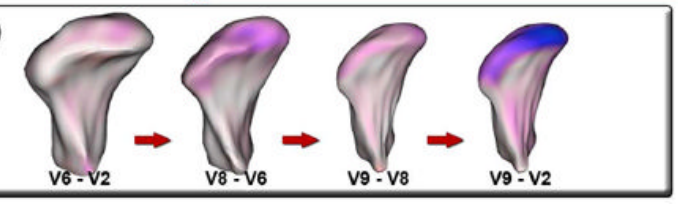

Figure 9.

Close up views of the longitudinal follow-up of changes in the right condyle in figure 9 . The findings for this patient reveal bone resorptive changes approximately four times larger than other cases in our TMJ OA asymptomatic database (data not included on this paper). A. Frontal view of condylar morphology prior to surgery, at splint removal, 1 year postsurgery and 2 years postsurgery. B-E. Absolute distances and difference vectors B. Presurgery subtracted from splint removal. C. Splint removal subtracted from 1 year postsurgery; D. One year postsurgery subtracted from 2 years postsurgery; E. Presurgery subtracted from 2 years post surgery; F. Visualization of changes using signed distances, where bone formation is shown in red, $5 \mathrm{~mm}$ of bone resorption in the superior and articular surfaces in blue. 
A)
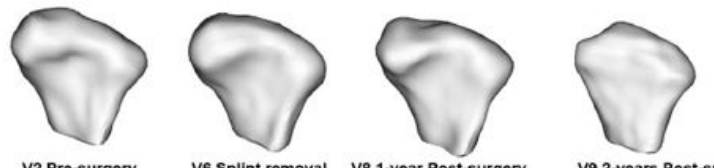

v6 Splint removal V8 1-year Post-surgery
V9 2-years Post-surgery
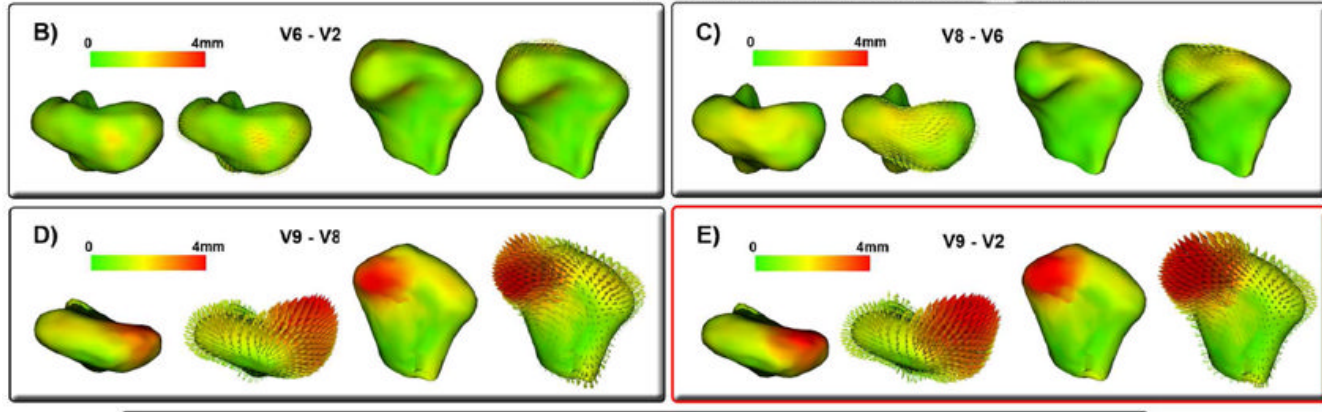

E) $\quad 0 \quad 4 \mathrm{~mm} \quad$ V9 - V2
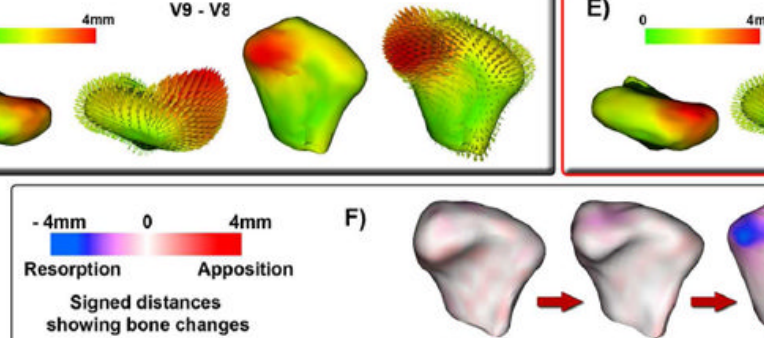

F)
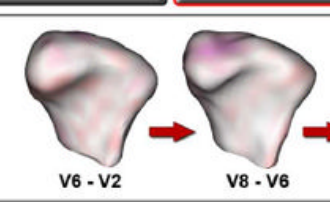

v8 - v6
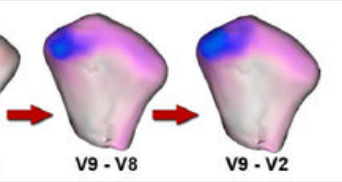

Figure 10.

Close up views of the longitudinal follow-up of changes in the left condyle in figure 10. The findings for this patient reveal bone resorptive changes approximately four times larger than other cases in our TMJ OA asymptomatic database (data not included on this paper). A. Frontal view of condylar morphology prior to surgery, at splint removal, 1 year postsurgery and 2 years postsurgery. B-E. Absolute distances and difference vectors B. Presurgery subtracted from splint removal. C. Splint removal subtracted from 1 year postsurgery; D. One year postsurgery subtracted from 2 years postsurgery; E. Presurgery subtracted from 2 years postsurgery F. Visualization of changes using signed distances, where bone formation is shown in red, $4 \mathrm{~mm}$ of bone resorption in the superior and articular surfaces in blue. 


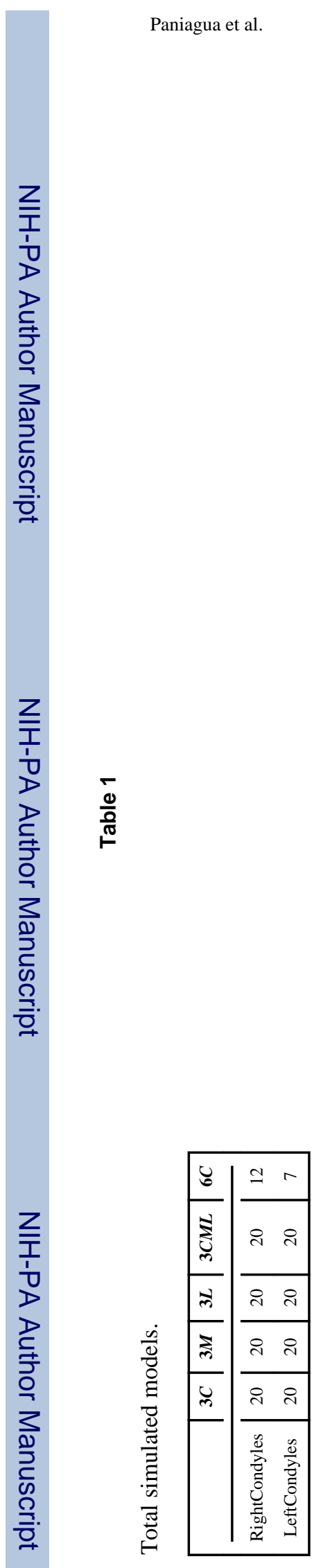

Page 20

\begin{tabular}{|c|c|}
\hline U్ & $\simeq r$ \\
\hline 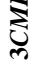 & ते त \\
\hline ले & ¿ \\
\hline ले & ते तి \\
\hline ల్ల & ¿ त \\
\hline & \\
\hline & 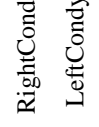 \\
\hline
\end{tabular}

Comput Med Imaging Graph. Author manuscript; available in PMC 2012 July 1. 


\begin{tabular}{|c|c|c|c|c|c|}
\hline U్ర & $\begin{array}{l}\infty \\
\text { ò } \\
0\end{array}$ & 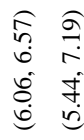 & U & $\hat{\sigma}$ & 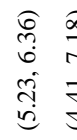 \\
\hline 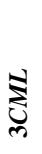 & ठे & 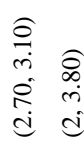 & : & $\underset{-}{\&}$ & 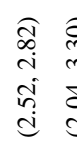 \\
\hline ले & $\stackrel{\&}{\stackrel{\leftrightarrow}{-}}$ & 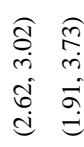 & $\vec{m}$ & ô & 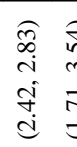 \\
\hline ले & $\stackrel{8}{-}$ & 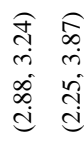 & ঙ & o. & 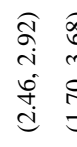 \\
\hline ల్ల & ò & 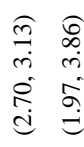 & $\bigcup_{n}$ & $\underset{-}{8}$ & 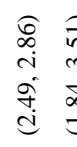 \\
\hline 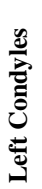 & $\begin{array}{l}n \\
o \\
v \\
\frac{n}{2} \\
1 \\
1 \\
\frac{1}{2} \\
2\end{array}$ & 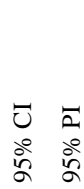 & 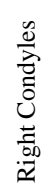 & $\begin{array}{l}n \\
o \\
v \\
\text { sa } \\
1 \\
\frac{1}{2} \\
\frac{1}{2}\end{array}$ & 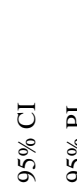 \\
\hline
\end{tabular}

Comput Med Imaging Graph. Author manuscript; available in PMC 2012 July 1. 
Table 3

Statistical results based on the defects.

\begin{tabular}{|l|c|c|}
\hline Defects & 3mm Avg & 6mm Avg \\
\hline$P\left(\left|X-\mu_{0}\right|\right)<0.5$ & 1 & 1 \\
$95 \% \mathrm{CI}$ & $(2.66,2.91)$ & $(5.99,6.35)$ \\
$95 \% \mathrm{PI}$ & $(2.22,3.36)$ & $(5.53,6.81)$ \\
\hline
\end{tabular}

